\title{
Bandwidth Utilization Optimization on Agent Based CR Networks
}

\author{
Aladje Umaro Balde ${ }^{1}$, Cui Lin ${ }^{2}$ \\ ${ }^{1,2}$ Tianjin University of Technology and Education, School of Information Technology and Engineering, Add: Lin liu East, Hexi District, \\ Tianjin, P.R.C
}

\begin{abstract}
Cognitive radio (CR) increases spectrum efficiency by allowing secondary users (SUs) to dynamically use the idle spectrum owned by primary users (PUs). This paper studies bandwidth allocation of SUs for throughput efficiency. Taking on consideration a scenario of five nodes on which two of them are Primary Users(PUs) and the other three are secondary users (SUs). What amount of bandwidth should be leased to the secondary users? In this paper, we study the optimal bandwidth allocation for multiple SUs. Our approach addresses the transmission parameters both in Primary Users (PUs) and Secondary Users (SUs). We first analyze the amount of bandwidth used by the Primary Users (Pus) and design an algorithm that will distribute the remaining bandwidth and allocate the optimal bandwidth an SU should use to maximize the per-SU throughput in the long term. We adjust the Secondary users TCP parameters so as it should not use more than the allocated Bandwidth to improve SUs' performance and also propose the algorithm for bandwidth sharing among the Pus and SUs more efficient while taking on account for the dynamics of the SUs on the idle bandwidth exploitation. We use the NS2 cognitive radio simulations to validate our results. The work contribute and steer the initiatives of the researches on finding the adequate bandwidth sharing algorithm in cognitive radio networks.
\end{abstract}

Keywords: NS2, Cognitive Network (CR), Primary User (Pu) and Secondary User (Su).

\section{Introduction}

Research in Cognitive radio networks recently has attracted many researchers, this is due to the importance of the cognitive radio in utilization of the available communication channels. Cognitive Radio networks are the ones in which the licensed frequency channels used by the licensed users can be used by the unlicensed users when they became available. Cognitive Radio can capture or sense temporal and spatial variations in the radio environment, allowing it to find unoccupied portions of spectrum in real time. To exploit these holes in spectrum activity, Cognitive radio is also capable of dynamically adjusting operating frequency, bandwidth, and other physical layers parameters[1], [2], [3]. The spectrum sharing can be achieved through two ways which are spectrum overlay (Time division Multiplexing) and Spectrum underlay (Frequency division multiplexing).Spectrum underlay in cognitive radio allows primary users and secondary users to transmit simultaneously provided that the interference power received by the primary user is below the prescribed threshold [4]. In overlay systems the secondary users overhear the transmission of the primary users, then use this information along with sophisticated signal processing and coding techniques to maintain or improve the performance [5].Cognitive Radio deployment poses some challenges due various dynamics in it. The major challenge is to efficiently use the spectrum opportunities due to primary user activity while protecting the performance of primary users [1]. In a scenario of two primary users and three secondary users the bandwidth allocation becomes complex, also managing the handovers between the primary users and secondary users. This paper contribute the algorithm to handle the bandwidth sharing between the primary users and secondary users in a Cognitive Radio Network. This paper is organized in four parts Introduction, System setup, Simulation results and Conclusion.

\section{System Setup}

Network simulator 2 is a networking simulator based on TCL scripting language. OTCL provides the objects in NS2 simulator, some classes are implement using the $\mathrm{C}++$ but they are interfaced with the help of the OTCL. Ns is a discrete event simulator targeted at networking research. Ns provides substantial support of TCP, routing and multicast protocols over wired and wireless (local and satellite) networks[6]. The scenario is simulated with the use of the wireless nodes in Ns2. Two of the wireless nodes acts as the Primary users (Pus) and other three acts as the Secondary users (Sus). The transmission range of the nodes is enhanced to $250 \mathrm{M}$ so as we can have the nodes communicate even when they are not too close. On data transmission, the Ns2 agents are deployed to which the TCP and UDP agents acts as data sources and the Null and Sink agents used to discard the sent packets at the other end of the data transmission. The ns-2 simulation environment offers great flexibility in investigating the characteristics of the wireless networks because it already contains flexible models energy constrained wireless ad hoc networks. 
International Journal of Science and Research (IJSR)

ISSN (Online): 2319-7064

Index Copernicus Value (2013): 6.14 | Impact Factor (2014): 5.611

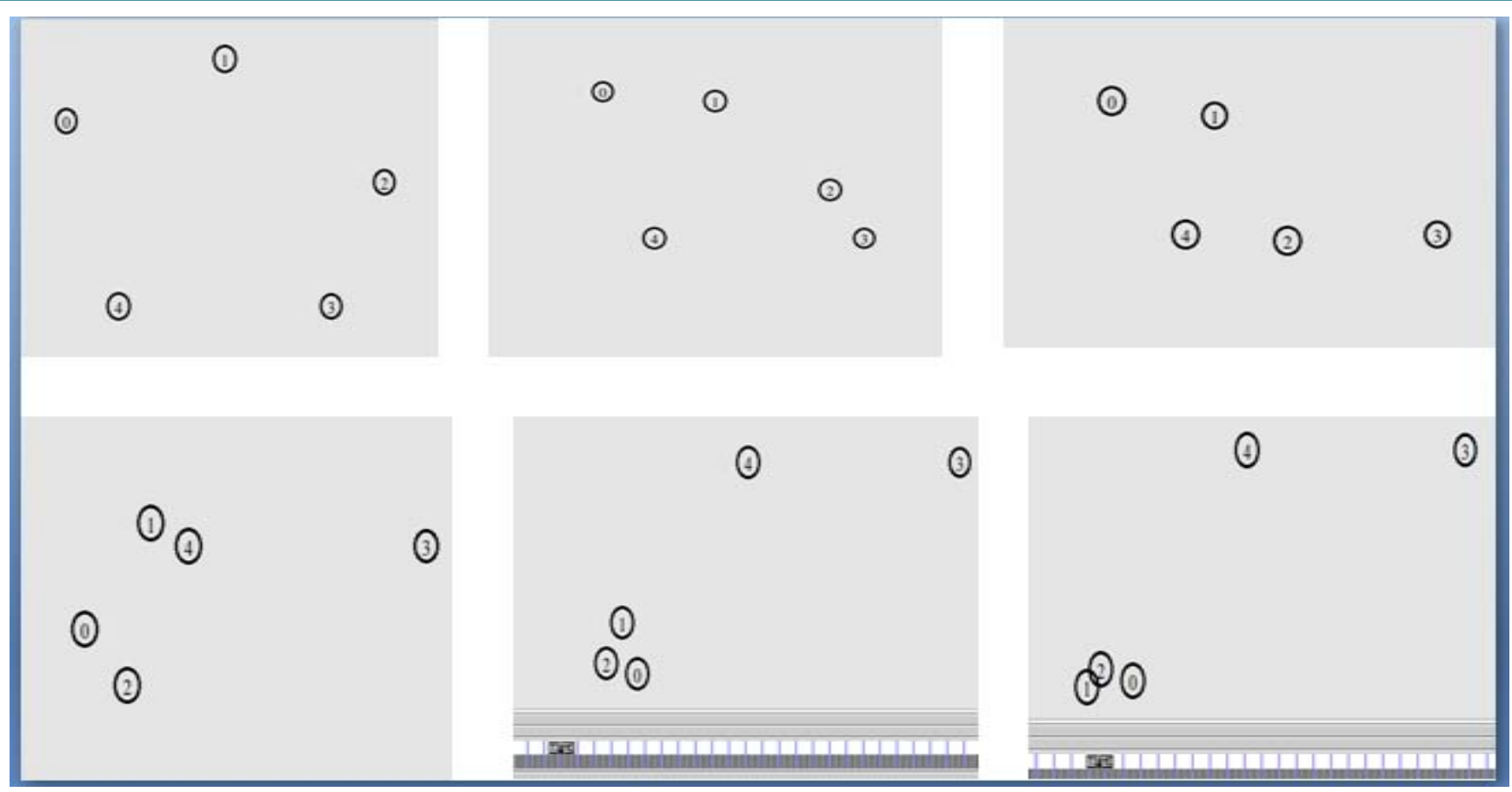

Figure 2a

\section{Methodology}

The goal of achieving Secondary users to exploit the available bandwidth in the primary users transmission channels without deteriorating the primary users (Pus) performance is experimented in this paper. There two main objectives to be experimented

1) The algorithm that is carried out in order to smoothly allocate the available bandwidth to the secondary users without diminishing the primary users (Pus) performance.

2) The formula used to estimate the optimal bandwidth that can be allocated to a secondary users (Sus) without causing performance issues to the primary users (Pus).

In practical multiuser environments, cognitive radio operation is governed by interference tolerance and sensing limits at the primary and secondary users. The interference limits at the primary and secondary users indicate the amount of protection needed at each (primary or secondary) user from the multiuser interference to maintain a certain rate [7]. The important challenge is how many secondary users and primary users can share the system for better throughput? , in that system what is the optimal amount of bandwidth is safe to be shared between the secondary users without degrading the system performance? This is reminiscent of the familiar debate of licensing versus autonomy, a tradeoff that is fundamental to many areas of systems and control theory [7]. In a cognitive radio system consisting of $\mathrm{N}$ secondary users and one primary user, a primary user using the spectrum of bandwidth $\mathrm{W}$ and the bandwidth is shared among the $\mathrm{n}$ secondary users. Denote the instantaneous channel gains between the $i^{\text {th }}$ SU transmitter (SU-Tx) and the $i^{\text {th }}$ SU receiver (SU-Rx) and between the $\mathrm{i}^{\text {th }}$ (SU-Tx) and the PU receiver (PU-Rx) by hi and $\mathrm{g}_{\mathrm{i}}$, respectively. Then the corresponding instantaneous channel gain vectors are g $\triangleq$ $\left[\mathrm{g} 1 \mathrm{~g} 2 \cdots \mathrm{g}_{\mathrm{N}}\right]$ and $\mathrm{h} \triangleq\left[\mathrm{h}_{1} \mathrm{~h}_{2} \cdots \mathrm{h}_{\mathrm{N}}\right]$, and they represent particular realizations of the channel fading vector random processes $\mathrm{G}$ and $\mathrm{H}$, respectively, which are assumed to be ergodic and stationary [4].Then the optimal bandwidth allocation is

$w_{i}^{*}(\mathrm{~h}, \mathrm{~g})=\mathrm{W} h_{i} p_{i}^{*}(\mathrm{~h}, \mathrm{~g}) / \sum_{i=1}^{N} h_{i} p_{i}^{*}(\mathrm{~h}, \mathrm{~g})$.

Apart from having an optimal bandwidth allocation formula, we need an adequate algorithm to take in charge of the system during system operations, a system having $\mathrm{n}$ numbers of secondary users and in a consecutive manner they try to access the available bandwidth hence there is a need for checking if the channel has a bandwidth to share before even evaluating what bandwidth can be shared. In this regard we need to always evaluate the system for the availability of the bandwidth and store that value for future reference at the time when the secondary user need to get a share from it. The following is the flow chart of the Bandwidth allocation steps to the Secondary user in a cognitive radio system that is experiment
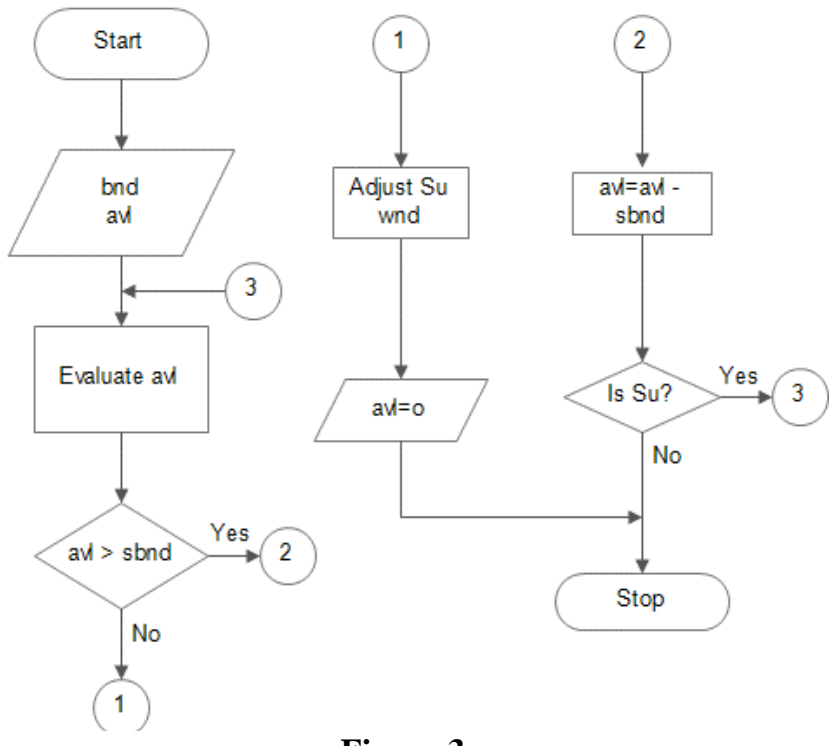

Figure 3a 


\section{International Journal of Science and Research (IJSR) \\ ISSN (Online): 2319-7064}

Index Copernicus Value (2013): 6.14 | Impact Factor (2014): 5.611

\section{Result and Discussion}

The Cognitive radio system is set up with a $10 \mathrm{M}$ bandwidth to be shared among the system participants, the simulation time was set to $10 \mathrm{sec}$. Fig $4 \mathrm{a}$ shows the Primary user $(\mathrm{Pu})$ transferring the data alone and is not exploiting all the available bandwidth hence allowing the possibility of the secondary users to exploit the remaining unused bandwidth. Using the algorithm we orderly manage the bandwidth allocation puzzle and use the optimal bandwidth allocation formula to allocate the bandwidth to the secondary users available, in case where the secondary users TCP window were is larger than the allocated bandwidth we adjust the window in order to efficiently and smoothly transfer the data. Fig $4 \mathrm{~b}$ shows the bandwidth sharing among a primary user and a secondary user in a smooth way without causing interference to the primary user.

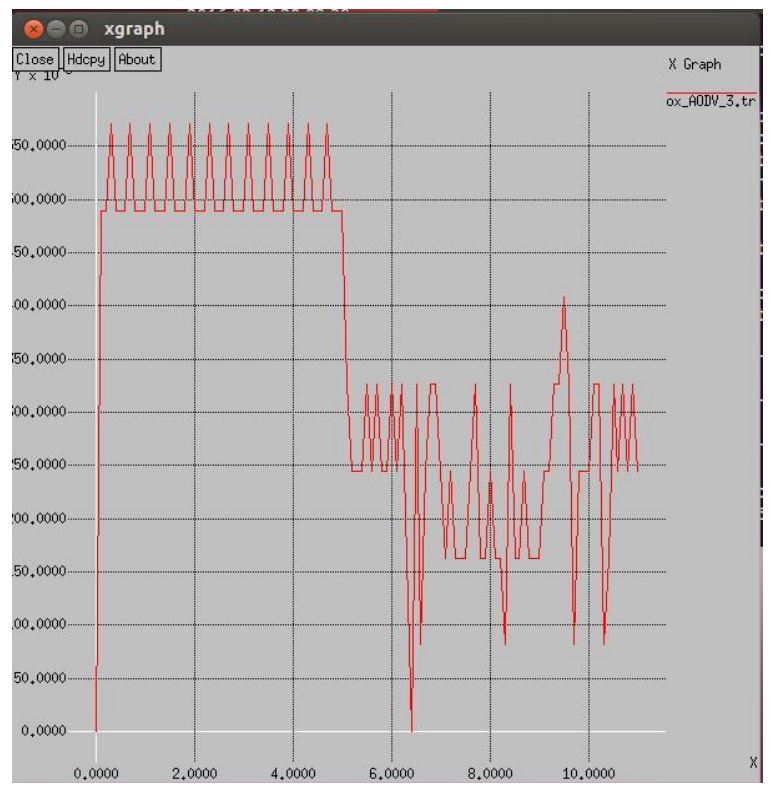

Figure 4a

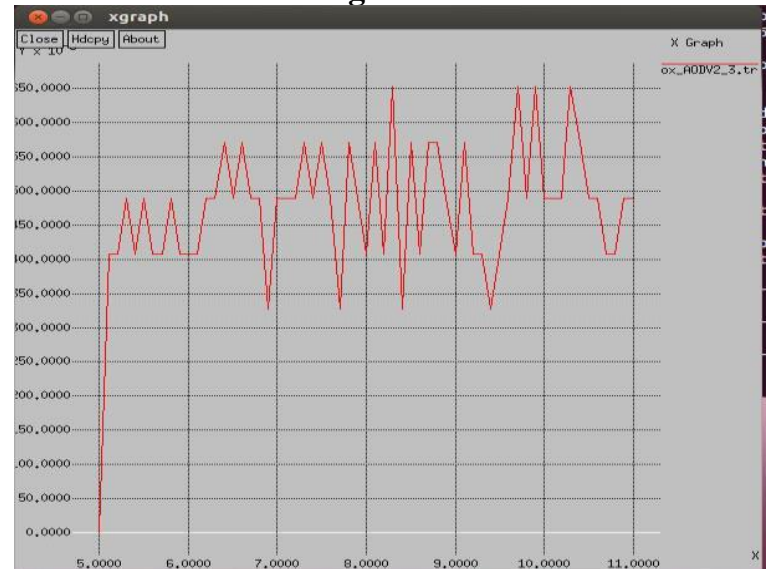

Figure 4b

\section{Related Works}

Bandwidth allocation in Agent based Cognitive radio networks attracts many researchers due to its promising solution on efficiently utilization of the licensed spectrum. An algorithm has been proposed in [8] to improve the utilization of bandwidth in agent based cognitive radio networks, the algorithm contributes much on the cost of the of the agent on leasing the bandwidth obtained from the primary user to the secondary users, it did not adequately address the issues of the optimal bandwidth to secondary users for an efficient agent based cognitive radio networks. The researchers in [9] have used game theory to solve the puzzle of resource allocation on cognitive radio networks to which they extend their solution to multi agent cognitive radio networks. In [10] they compared single agent based and multi agent based cognitive radio networks and their advantages and disadvantages on the secondary user autonomy on exploiting the available bandwidth.

\section{Conclusion}

Bandwidth allocation on agent based cognitive radio networks is one of the most important parameters to make the system operate more smoothly, an efficient algorithm is a vital component on allowing the secondary users to participate on the system and prevent distortion on the primary user spectrum utilization. The proposed serves this purpose and with the help of the optimal bandwidth formula we have obtained promising results.

\section{References}

[1] Danxu, eajung, xinliu "Optimal Bandwidth selection in multi channel Cognitive radio networks: how much is too much?"

[2] IEEE 802.22 WRAN WG, www.ieee802.org/22/.

[3] S. Haykin. Cognitive radio: Brain-empowered wireless communications. IEEE Journal on Selected Areas in Communications., 23(2):201-220,Febraury 2005.

[4] Xiaowen Gong, Sergiy A. Vorobyov, and Chintha Tellambura "Joint Bandwidth and Power allocation in cognitive Radio under fading channels" ICASSP 2011.

[5] Andrea Goldsmith and Ivan Maric "Capacity of Cognitive Radio Networks"

[6] Network simulator "http://www.isi.edu/nsnam/ns/"

[7] Sudhir Srinivasa and Syed Ali Jafar "Cognitive Radio Networks: How much Spectrum Sharing is Optimal?",Download available at : http://citeseerx.ist.psu.edu/viewdoc/download?doi=10.1. 1.565.323\&rep=rep $1 \&$ type $=$ pdf

[8] Tian Chu, Peng Cheng, Lin Gao, Xinbing Wang, Hui Yu, Xiaoying Gan "Spectrum Trading in Cognitive Radio Network: AnAgent-based Model under Demand Uncertainty"

[9] ASMA AMRAOUI ${ }^{1}$, BADR BENMAMMAR ${ }^{1}$, FRANCINE KRIEF “"Cognitive Radio Resource Management Using Multi-Agent Systems, Auctions and Game Theory", WSEAS TRANSACTIONS on COMPUTERS, Volume 13, 2014.

[10] Emna Trigui, Moez Esseghir and Leila Merghem Boulahia“ON USING MULTI AGENT SYSTEMS IN COGNITIVERADIO NETWORKS: A SURVEY", International Journal of Wireless \& Mobile Networks (IJWMN) Vol. 4, No. 6, December 2012. 


\section{International Journal of Science and Research (IJSR) \\ ISSN (Online): 2319-7064}

Index Copernicus Value (2013): 6.14 | Impact Factor (2014): 5.611

\section{Author Profile}

Aladje umaro Balde received the Bachelor Degree in computer science from Tianjin University of Technology and Education (China) in 2007 - 2012, respectively. During $2007-2008$ he did one year Chinese language. In 2003-2004 he received a an Ordinary Diploma from the University of Amilcar Cabral (Guinea-Bissau). In 20022003 he was awarded a Diploma in Journalist from the Agency of Bissau Media and Publications (Guinea- Bissau). My research areas are in Cognitive Radio networks, Wireless Adhoc networks and Satellite network. 